\title{
CrystEngComm
}

Check for updates

Cite this: CrystEngComm, 2018, 20 6458

Received 29th July 2018,

Accepted 14th September 2018

DOI: $10.1039 /$ c8ce01264d

rsc.li/crystengcomm

\section{Graphene-like metal-organic frameworks: morphology control, optimization of thin film electrical conductivity and fast sensing applications $\dagger$}

\author{
Bastian Hoppe, (D) *ad Karen D. J. Hindricks, ${ }^{\text {*ad }}$ Dawid P. Warwas, ${ }^{\text {aad }}$ \\ Hendrik A. Schulze, ${ }^{\text {ad }}$ Alexander Mohmeyer, ${ }^{\text {ad }}$ Tim J. Pinkvos, ${ }^{\text {ad }}$ \\ Saskia Zailskas, ${ }^{\text {ad }}$ Marc R. Krey, ${ }^{\text {ad }}$ Christopher Belke, ${ }^{\text {bd }}$ Sandra König, ${ }^{c}$ \\ Michael Fröba, iD ${ }^{c}$ Rolf J. Haug (iD*bd and Peter Behrens (iD *ad
}

\begin{abstract}
The metal-organic framework $\mathrm{Cu}-2,3,6,7,10,11$-hexahydroxytriphenylene ( $\mathrm{Cu}_{3} h$ htp $\mathrm{p}_{2}$-MOF), a copper-based graphene-like framework, is one of the few MOFs featuring inherent electrical conductivity. Here, we investigate the synthesis of this material with regard to the influence of different additives. It is shown that ammonia acts as a modulator leading to platelet-like particles in a water-based synthesis system. This material is thoroughly characterized by X-ray diffraction (XRD), electron microscopy, atomic force microscopy (AFM), physisorption, thermal behaviour, and electrical conductivity. The measured conductivity value of $0.045 \mathrm{~S} \mathrm{~cm}^{-1}$ surpasses all formerly reported measurements. The obtained platelets appear especially suitable for the preparation of different devices. As an example, we prepared thin and homogenous films by spray-coating water-based dispersions of this MOF on glass and on polymer substrates. In the films, the platelets are oriented parallel to the substrate and are in intimate contact. This leads to a high electrical conductivity combined with an easily accessible pore system. The applicability of such coatings is shown in a preliminary sensing test, showing quick and strong response and fast recovery. This work shows that control of the crystal morphology combined with suitable preparation procedures can enhance the performance of MOF-based devices.
\end{abstract}

\section{Introduction}

In recent years, the research on metal-organic frameworks (MOFs) has grown tremendously due to their permanent porosity and versatile opportunities to manipulate their properties (e.g. surface area, pore size, functional groups and active sites for catalysis) for different potential applications like gas storage and separation, catalysis, sensing or in biomedical applications. However, despite the large variety of MOFs described, members of this family with inherent electrical conductivity are extremely rare. To overcome this lack, nonconductive MOFs can be infiltrated by conjugated organic

\footnotetext{
${ }^{a}$ Institut für Anorganische Chemie, Leibniz Universität Hannover, Schneiderberg 39, Callinstraße 9, 30167 Hannover, Germany. E-mail: peter.behrens@acb.uni-hannover.de ${ }^{b}$ Institut für Festkörperphysik, Leibniz Universität Hannover, Schneiderberg 39, Appelstraße 2, 30167 Hannover, Germany

${ }^{c}$ Institut für Anorganische und Angewandte Chemie, Universität Hamburg, MartinLuther-King-Platz 6, 20146 Hamburg, Germany

${ }^{d}$ Laboratory of Nano and Quantum Engineering (LNQE), Leibniz Universität

Hannover, Schneiderberg 39, 30167 Hannover, Germany

$\dagger$ Electronic supplementary information (ESI) available. See DOI: 10.1039/ c8ce01264d
}

molecules like tetracyanoquinodimethane (TCNQ) ${ }^{1}$ or conductive organic polymers ${ }^{2}$ and thereby attain electrical conductivity. Unfortunately, this procedure reduces the accessible pore volume, and is thus detrimental to the combination of efficient sorption properties and electrical conduction.

Recently, a new group of graphene-like 2D metal-organic frameworks has been reported which exhibits inherent high electrical conductivity of the metal-organic structure and an open pore system at the same time. These 2D MOFs are built up from phenylene ${ }^{3-5}$ or triphenylene ${ }^{5-11}$ linker molecules with six functional groups $\left(-\mathrm{OH},-\mathrm{NH}_{2}\right.$ or $\left.-\mathrm{SH}\right)$. Based on the trigonal linker molecules and square-planarly coordinated metal ions (e.g. $\left.\mathrm{Cu}^{2+}, \mathrm{Ni}^{2+}, \mathrm{Co}^{2+}, \mathrm{Pt}^{2+}\right)$, layers with a hexagonal structure are formed which topologically resemble that of graphene on a larger scale. These layers can stack to build up a one-dimensional pore system. Members of this group of graphene-like MOFs possess electrical conductivities ranging from $0.002-58.8 \mathrm{~S} \mathrm{~cm}^{-1}$ (pressed pellets) ${ }^{6,12}$ at room temperature. Thin films of $\mathrm{Cu}_{3} b h t_{2}$ (benzenehexathiol, bht) with a thickness of $400 \mathrm{~nm}$ show even higher values up to $1580 \mathrm{~S}$ $\mathrm{cm}^{-1}$. ${ }^{4}$ However, this MOF is not porous because the specific stacking of the layers leads to pore blocking. Electrodes built 
from graphene-like MOFs have been tested for hydrogen evolution from water, ${ }^{5,13,14}$ oxygen reduction reactions, ${ }^{15}$ sensing of volatile organic compounds (VOCs) ${ }^{6,7}$ and for energy storage in supercapacitors. ${ }^{9}$

The electronic structure of $\mathrm{Ni}_{3}$ hitp 2 (hexaiminotriphenylene, hitp) has been investigated by density functional theory (DFT) and $a b$ initio calculations, stating that a monolayer of this MOF is semiconductive with bandgaps between $0.13 \mathrm{eV}$ (ref. 16 and 17) and $0.25 \mathrm{eV} .{ }^{18}$ When a second layer is added to the simulation model, the bandgap decreases with the interlayer distance. For a slipped parallel stacking the band gap disappears at $3.8 \AA$ A. The bulk material with an experimentally determined interlayer distance of $3.3 \AA$ does not have a bandgap and the material shows metallic conductivity. Consequently, this material should have an appreciable electrical conductivity not only parallel but also perpendicular to the stacked layers of the MOF. ${ }^{18}$ In contrast to the nickelbased MOF, not only the bulk material but also a monolayer of $\mathrm{Cu}_{3}$ hitp 2 is predicted to be a metallic conductor without a bandgap. $^{17}$

In a final device, cooperative properties like electrical conductivity depend not only on the actual crystal and electronic structure of a compound, but also on defects, (anisotropy of) crystal morphology, and the packing and orientation of primary crystals. Thus, for generating favourable effects, the fabrication of crystal morphologies suitable for a certain fabrication procedure ("shaping") is also of prime importance. In case of the combined application of nanoporosity and electrical conductivity, this is especially important, e.g., with respect to good contacts and favourable orientations at the crystal boundaries as well as with respect to the accessibility of the pore system and short diffusion pathways. In this respect, it remains to be noted that in most of the aforementioned studies on graphene-like MOFs, no special significance was given to control the crystal morphology, the arrangement of crystals or the fabrication of shapes suitable for device applications. In this study, we describe the synthesis of thin platelets of the graphene-like MOF copper-2,3,6,7,19,11-hexahydroxytriphenylene $\left(\mathrm{Cu}_{3} h h t p_{2}\right)$ and the fabrication of highly homogeneous thin conducting films from stable dispersions of this material by spray-coating.

$\mathrm{Cu}_{3} h h t p_{2}$ shows the lowest conductivity values $(0.002 \mathrm{~S}$ $\mathrm{cm}^{-1}$, pressed pellet; ${ }^{6} 0.001-0.02 \mathrm{~S} \mathrm{~cm}^{-1}$, thin film; ${ }^{19,20} 0.2 \mathrm{~S}$ $\mathrm{cm}^{-1}$, single crystal ${ }^{11}$ ) among the graphene-like MOFs, but it is the one which shows the strongest response towards many VOCs in sensing experiments. ${ }^{6}$ The reported synthesis by Yaghi and coworkers leads to rod-like particles within which the one-dimensional pores run along the particle elongation, an unfavourable situation with regard to the accessibility of the pore system. ${ }^{11}$ Dincă and coworkers explicitly resigned to check the influence of the particle morphology in their sensing experiments, although they found significant differences in crystal size and morphology between $\mathrm{Cu}_{3} h h t p_{2}, \mathrm{Cu}_{3} h i t p_{2}$ and $\mathrm{Ni}_{3}$ hitp ${ }_{2}{ }^{6}$ Recently reported investigations on $\mathrm{Cu}_{3} h i b_{2}$ and $\mathrm{Ni}_{3} h i b_{2}$ (hexaiminobenzol, hib) demonstrate that reaction conditions and postsynthetic treatments can have a strong impact on the properties of these materials. Whereas waterbased synthesis ${ }^{21}$ results in products with a BET surface area of about $350 \mathrm{~m}^{2} \mathrm{~g}^{-1}$ and a conductivity $0.7 \mathrm{~S} \mathrm{~cm}^{-1}\left(\mathrm{Ni}_{3} h i b_{2}\right)$ or $0.1 \mathrm{~S} \mathrm{~cm}^{-1}\left(\mathrm{Cu}_{3} h i b_{2}\right)$, the products from a DMSO-based synthesis $^{22}$ show lower BET surface areas but enhanced conductivities of $8 \mathrm{~S} \mathrm{~cm}^{-1}\left(151 \mathrm{~m}^{2} \mathrm{~g}^{-1}, \mathrm{Ni}_{3} h i b_{2}\right)$ and $13 \mathrm{~S} \mathrm{~cm}^{-1}(114$ $\left.\mathrm{m}^{2} \mathrm{~g}^{-1}, \mathrm{Cu}_{3} h i b_{2}\right)$.

The concept of modulated synthesis is a strong tool in MOF synthesis, especially with regard to influencing size and shape of MOF crystals. ${ }^{23-25}$ Within the concept of coordination modulation, modulators are molecules with only one coordinating function. They compete with linker molecules for the coordination sites at the metal cations and thus in general slow down nucleation and growth of the crystals, often leading to crystals of high quality. ${ }^{26}$ Especially the synthesis of large single crystals for the determination of new crystal structures often requires the presence of a modulator within the synthesis mixture. ${ }^{27,28}$ In some cases, crystalline products can only be obtained when a modulator is used during the synthesis. ${ }^{27,29,30}$ Also thermodynamically unfavourable phases, e.g. ZIF-71 and [Zn(dcim) $)_{2}$, can become accessible by the use of a modulator, ${ }^{31,32}$ although in these systems protonation modulation also plays a role for nanocrystal synthesis. $^{33}$

Here, we report on the control of crystal morphology of $\mathrm{Cu}_{3} h h t p_{2}$ by modulated synthesis. We tested different additives and found that by the addition of pyridine or ammonia accompanied by a change of the copper salt employed, we are able to synthesize large flake-like particles of this porous and conductive MOF. The flakes or platelets produced present their pore openings on their large surfaces, as the pores run vertically to the platelet plane. Thus, they should be very suitable for applications where fast diffusion into the pore system is necessary, for example with regard to the response time of sensors. We prepared water-based dispersions of the $\mathrm{Cu}_{3} h h t p_{2}$ platelets and used these to fabricate homogeneous thin films on glass and also flexible polycarbonate foils by simple spray-coating. In contrast to recent reports of thin film preparation by layer-by-layer spray-coating ${ }^{19}$ or the Langmuir-Blodgett transfer technique, ${ }^{20}$ our approach is easily adjustable for patterned deposition of small structures and conductor paths as well as for large area deposition. Also, the preferred flake-like morphology leads to highly textured orientation of the particles inside the film. All in all, this work demonstrates that detailed studies of MOF synthesis systems can lead to defined and uniform crystal morphologies, and that suitable morphologies can be beneficially used to fabricate well-defined shapes for potential applications.

\section{Experimental}

\subsection{Synthesis procedures}

2.1.1 Materials. Chemicals were purchased from commercial suppliers and used as received: copper(II) nitrate trihydrate $\left(\mathrm{Cu}\left(\mathrm{NO}_{3}\right)_{2} \cdot 3 \mathrm{H}_{2} \mathrm{O}\right.$, Sigma-Aldrich, $\left.\geq 99.5 \%\right)$, aqueous ammonia $\left(\mathrm{NH}_{4} \mathrm{OH}, \quad\right.$ Sigma-Aldrich, 28-30\% $\left.\mathrm{NH}_{3}\right)$, 
2,3,6,7,10,11-hexahydroxytriphenylene hydrate $\left(\mathrm{H}_{6} h h t p h\right.$, Tokyo Chemical Industry, >95\%), Span ${ }^{\circledR} 80$ (for synthesis, Merck), 1-butanol (Sigma-Aldrich, $\geq 99.4 \%$ ), 2-propanol (Sigma-Aldrich, $\geq 99.5 \%$ ), methanol (Sigma-Aldrich, $\geq 99.8 \%$ ), $N, N$-dimethylformamide (DMF, Sigma-Aldrich, $\geq 99.8 \%$ ), tetrahydrofuran (THF, Sigma-Aldrich, $\geq 99.9 \%$ ), pyrazole (SigmaAldrich, 98\%), pyridine (Sigma-Aldrich, $\geq 99 \%$ ), 2-propylamine (Sigma-Aldrich, 99.5\%), 1-propylamine (Fluka Analytical, $\geq 99 \%$ ), ethanol (VWR chemicals, absolute), APEC® polycarbonate foil (PC foil, Covestro).

2.1.2 Synthesis of $\mathrm{Cu}_{3} h \boldsymbol{h} t p_{2}$ for screening of additives. To investigate the influence of different additives on crystal growth, 10-100 eq. of a modulator was added to a dispersion of $51 \mathrm{mg} \quad(0.15 \mathrm{mmol}, 1 \quad$ eq. $) \quad 2,3,6,7,10,11-$ hexahydroxytriphenylene hydrate $\left(\mathrm{H}_{6} h h t p h\right)$ in $2.4 \mathrm{~mL}(144$ mmol, 962 eq.) dist. $\mathrm{H}_{2} \mathrm{O}$. Then a solution of $63 \mathrm{mg}(0.26$ mmol, 1.75 eq.) $\mathrm{Cu}\left(\mathrm{NO}_{3}\right)_{2} \cdot 3 \mathrm{H}_{2} \mathrm{O}$ in $2.4 \mathrm{ml}$ (144 mmol, 962 eq.) dist. $\mathrm{H}_{2} \mathrm{O}$ was added and the reaction vessel was placed in a pre-heated oven at $80{ }^{\circ} \mathrm{C}$ for $24 \mathrm{~h}$. After cooling to room temperature, the dark blue precipitate was collected by centrifugation und washed two times with $15 \mathrm{~mL}$ absolute ethanol. The product was then dried at room temperature. For purification the powders were extracted with absolute ethanol for $24 \mathrm{~h}$ in a Soxhlet apparatus and dried in vacuum at room temperature.

2.1.3 Synthesis of flake-like $\mathrm{Cu}_{3} h h t p_{2}$ nanoparticles. To a solution of $127 \mathrm{mg}$ ( $0.53 \mathrm{mmol}, 1.75$ eq.) $\mathrm{Cu}\left(\mathrm{NO}_{3}\right)_{2} \cdot 3 \mathrm{H}_{2} \mathrm{O}$ in 2 $\mathrm{ml}$ (111 mmol, 370 eq.) dist. $\mathrm{H}_{2} \mathrm{O}, 10,30$ or 50 eq. of conc. $\mathrm{NH}_{4} \mathrm{OH}$ solution was added. The dark blue solution was added to a dispersion of $103 \mathrm{mg}\left(0.30 \mathrm{mmol}, 1\right.$ eq.) $\mathrm{H}_{6} h \mathrm{htph}$ in $8.4 \mathrm{~mL}$ (466 mmol, 1555 eq.) dist. $\mathrm{H}_{2} \mathrm{O}$. The reaction vessel was sealed and placed in a pre-heated oven at $80^{\circ} \mathrm{C}$ for $24 \mathrm{~h}$. After cooling to room temperature, the dark blue precipitate was collected by centrifugation und washed two times with $15 \mathrm{~mL}$ absolute ethanol. The product was then dried at room temperature. For purification the powders were extracted with absolute ethanol for $24 \mathrm{~h}$ in a Soxhlet apparatus and dried in vacuum at room temperature.

2.1.4 Dispersions of $\mathrm{Cu}_{3} \boldsymbol{h} h \boldsymbol{t p}_{2}$ nanoparticles. For the preparation of water-based dispersions, it is preferential to use the still wet product from the ethanol washing/extraction procedure. This material (approximately $120 \mathrm{mg} \mathrm{Cu}_{3} h h t p_{2}$ ) is easily dispersed in $50 \mathrm{~mL}$ of dist. $\mathrm{H}_{2} \mathrm{O}$ by shaking. These dispersions $\left(2.4 \mathrm{mg} \mathrm{mL} \mathrm{mL}^{-1}\right)$ are very stable over weeks and can be used directly for spray-coating or other procedures.

2.1.5 Preparation of $\mathrm{Cu}_{3} h \boldsymbol{h t} \boldsymbol{p}_{2}$ films by spray-coating. Using the described dispersions, spray-coating was initially performed manually with a Sogolee AFC-101A airbrush. Prior to the coating, the glass substrates were cleaned with water and absolute ethanol and dried with a nitrogen stream. The coating was carried out with 30 repetitions at a substrate temperature of $60^{\circ} \mathrm{C}$.

For more controlled thin film generation on polycarbonate foils and glass substrates, an automated spray-coating robot from Walther Systemtechnik (Germersheim, Germany) was employed. The composition of the dispersion was adjusted by adding $2.6 \mathrm{~mL}$ of a $12 \mathrm{wt} \%$ ethanolic Span® 80 solution to the $\mathrm{Cu}_{3} h h t p_{2}$-dispersion and stirring for at least $1 \mathrm{~h}$. Prior to the coating, the polycarbonate foils were washed with absolute ethanol, dried with a nitrogen stream and fixed with an aluminium sample holder (see ESI $\dagger$ Fig. S1). The coating was carried out at a substrate temperature of $60^{\circ} \mathrm{C}$ and by equipping the spray-coating robot with an SMS-02 spraying valve to coat 5 samples simultaneously at the same time. The used vaporizing pressure was 1.2 bar and the nozzle was only slightly opened to deposit only a small amount of material at each of the 50-100 repetitions. For patterned depositions, masks with different structures were used under the same conditions (see ESI $\dagger$ Fig. S1). After spray-coating, the sample was washed cautiously with ethanol to remove the surfactant and dried in air afterwards.

\subsection{Structure simulation}

The simulation of the crystal structure was performed with BIOVIA Materials Studio 2017 (San Diego, USA). To construct the structure, a copper ion was coordinated in a squareplanar fashion with two linker molecules. This complex was then transferred into a unit cell with the space group P6/ $\mathrm{mmm}$ and a geometry optimization was performed. Subsequently, the cell parameters $a$ and $b$ were optimized by reference to the experimental reflections at 4.7, 9.5, 12.6 and $16.5^{\circ} 2 \theta$. Afterwards, the interlayer distance was optimised to $3.16 \AA$ according to the broad reflection at $28.2^{\circ} 2 \theta$. This procedure results in an AA stacking of the layered structure.

\subsection{X-ray diffraction}

Powder X-ray diffraction (XRD) patterns were measured in transmission using a STOE Stadi P diffractometer (Darmstadt, Germany) with $\mathrm{CuK}_{\alpha 1}$ radiation with a wavelength of $\lambda$ = 1.5406 $\AA$ and a Ge (111) monochromator. The powders were grinded before the measurement.

$\mathrm{X}$-ray diffraction patterns of coatings were measured in reflection using a STOE Stadi P diffractometer (Darmstadt, Germany) working in $\theta-\theta$ geometry with $\mathrm{CuK}_{\alpha}$ radiation with a wavelength of $\lambda=1.5418 \AA$ and a nickel foil in front of the detector.

\subsection{Scanning electron microscopy}

Low-magnification scanning electron microscopy (SEM), mainly used for particle morphology screening, was carried out with a JEOL JSM-6610LV (Tokyo, Japan) with a tungsten filament, a working distance of $10 \mathrm{~mm}$ and an acceleration voltage between 10 and $20 \mathrm{kV}$. The bulk material was dispersed in ethanol and dropped onto polished carbon. Highresolution SEM (HR-SEM) was carried out on a JEOL JSM6700F (Tokyo, Japan) with a field emission cathode, a working distance of $3 \mathrm{~mm}$ and an acceleration voltage of $2 \mathrm{kV}$. The diluted dispersions in water were dropped on acetonecleaned gold-sputtered silicon wafers. 


\subsection{Transmission electron microscopy}

Transmission electron microscopy (TEM) was performed using a FEI Tecnai G2 F20 TMP $\left(C_{\mathrm{S}}=2 \mathrm{~mm}, C_{\mathrm{C}}=2 \mathrm{~mm}\right.$, Hillsboro, USA) with a $200 \mathrm{kV}$ field emission gun. $10 \mu \mathrm{L}$ of diluted dispersions were drop-casted on Quantifoil 400 mesh carbon-coated copper grids (Jena, Germany) and images were taken in bright-field mode. Distances were measured with NIH ImageJ (Bethesda, USA).

\subsection{Atomic force microscopy}

For atomic force microscopy, $10 \mu \mathrm{L}$ of a diluted water-based dispersion was drop-casted on a silica-coated silicon wafer and spin-coated after a latency period of 20 minutes. The measurements were performed with a Veeco Dimension V (Plainview, USA) equipped with a NanoWorld Pointprobe silicon cantilever (Neuchâtel, Switzerland) in tapping mode. The measurement was performed with a scan size of $10 \mu \mathrm{m}$ at 0.5 $\mathrm{Hz}$ and a scan angle of $0^{\circ}$. Height determinations of individual particles were performed with the implemented software Nanoscope 7.30. Topographic images were prepared with Gwyddion 2.49 (Czech Metrology Institute, Brno).

\subsection{Confocal microscopy}

In addition to the SEM investigations, confocal microscopy is used for the determination of coating thickness as well as for the evaluation of coating quality. Topographical imaging was performed using a Leica DCM3D scanning confocal microscope (Wetzlar, Germany) and the associated software LeicaScan version 3.2.3. Overview images were taken in the microscopic mode at a tenfold magnification with white light. Using the confocal image and the surface topography mode, the $3 \mathrm{D}$ view of the surface was constructed at a fiftyfold magnification, utilizing the EPI50x 0.9-L lens. Images were taken over the desired distance within $z$-direction with a step size of $0.2 \mu \mathrm{m}$, starting at the bottom. A lower threshold of $2 \%$ was used. Topographic images and height distributions using one-dimensional statistic functions were prepared with Gwyddion 2.49 of the Czech Metrology Institute (Brno, Czech).

\subsection{Physisorption experiments}

Argon physisorption isotherms were measured with a Quantachrome Autosorb-1 (Odelzhausen, Germany). The samples were Soxhlet-extracted and activated prior to the measurement for $24 \mathrm{~h}$ at $80{ }^{\circ} \mathrm{C}$ in vacuum. For the determination of the BET specific surface area the "Micropore BET Assistant" implemented in the ASiQwin 2.0 software form Quantachrome was used. The pore size distribution was calculated by fitting the NLDFT Quantachrome kernel "Ar at $87 \mathrm{~K}$ zeolites/silica" to the experimental data.

Water physisorption isotherms were measured with a Quantachrome Aquadyne DVS-2 (Odelzhausen, Germany) at $25^{\circ} \mathrm{C}$. The samples were Soxhlet-extracted and activated prior to the measurement for $24 \mathrm{~h}$ at $80^{\circ} \mathrm{C}$ in a dry nitrogen

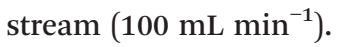

\subsection{Zeta potential measurement}

MOF dispersions (2.4 $\mathrm{mg} \mathrm{ml}^{-1}$ ) were characterized by $\mathrm{pH}^{-}$ dependent zeta potential measurements using a Malvern Zetasizer Nano ZS (Kassel, Germany) equipped with a Malvern multi purpose titrator MPT-2 (Kassel, Germany). 0.1 $\mathrm{M} \mathrm{HCl}$ and $\mathrm{NaOH}$ solutions were used to adjust the $\mathrm{pH}$ of the dispersion.

\subsection{X-ray photoelectron spectroscopy}

X-ray photoelectron spectroscopy measurements were performed with a Leybold Heraeus EA 10/100 (Cologne, Germany) spectrometer. A hemispheric analyzer with a diameter of $100 \mathrm{~mm}$ and non-monochromatic $\mathrm{MgK}_{\alpha}$ radiation of $1253.6 \mathrm{eV}$ was used for the measurement. The films were deposited on gold-coated silicon wafers using automated spraycoating with Span ${ }^{\circledR} 80$ as surfactant and were cleaned thoroughly by Soxhlet extraction with ethanol for 4 hours before the measurement.

\subsection{Thermal gravimetric analysis}

To investigate the thermal stability of the MOF, the simultaneous thermogravimetry and differential scanning calorimeter Netzsch STA 409 PC (Selb, Germany) was used. Experiments were performed in the range of $40-400{ }^{\circ} \mathrm{C}$ with a heating ramp of $5{ }^{\circ} \mathrm{C} \mathrm{min}^{-1}$ in an oxygen/argon (20/80) atmosphere. Furthermore, the device is coupled with a Netzsch QMS 403 D Aeolos quadrupole mass spectrometer (Selb, Germany) to obtain additional information on the combustion products.

\subsection{Measurements of electrical conductivity}

Conductivity measurements of pressed pellet samples were performed with the van der Pauw method using a Keithley 2100 multimeter (Beaverton, USA) and steel probes. With the van der Pauw method me $^{34,35}$ the resistances of contacts and wires are excluded and only the resistance of the material is observed. The samples were measured at room temperature under argon atmosphere in a glovebox after activation in vacuum for at least 24 hours. Pellets (diameter of $8 \mathrm{~mm}$, thickness between $0.13-0.41 \mathrm{~mm}$ ) were pressed using a hydraulic press for 1 minute at a weight of 1 ton.

Conductivity measurements of thin films were performed using a self-made linear 4-point-probe setup with a Keithley 2100 multimeter (Beaverton, USA). The samples were measured at room temperature under argon atmosphere in a glovebox after activation in vacuum for at least 24 hours.

\subsection{Sensing experiments}

With regard to a possible cross-sensitivity, investigations on the effect of water on the electrical conductivity of $\mathrm{Cu}_{3} h h t p_{2}$ were performed first. Pressed pellets were placed between 
two brass electrodes inside of a PTFE cell. The cell was placed in an argon-filled desiccator to avoid influences of moisture from the environment. A constant potential of $500 \mathrm{mV}$ was applied to the electrodes using an Ametek Princeton Applied Research Versastat 4 potentiostat (Berwyn, USA). Prior to the measurement the setup was flushed with dry argon. During
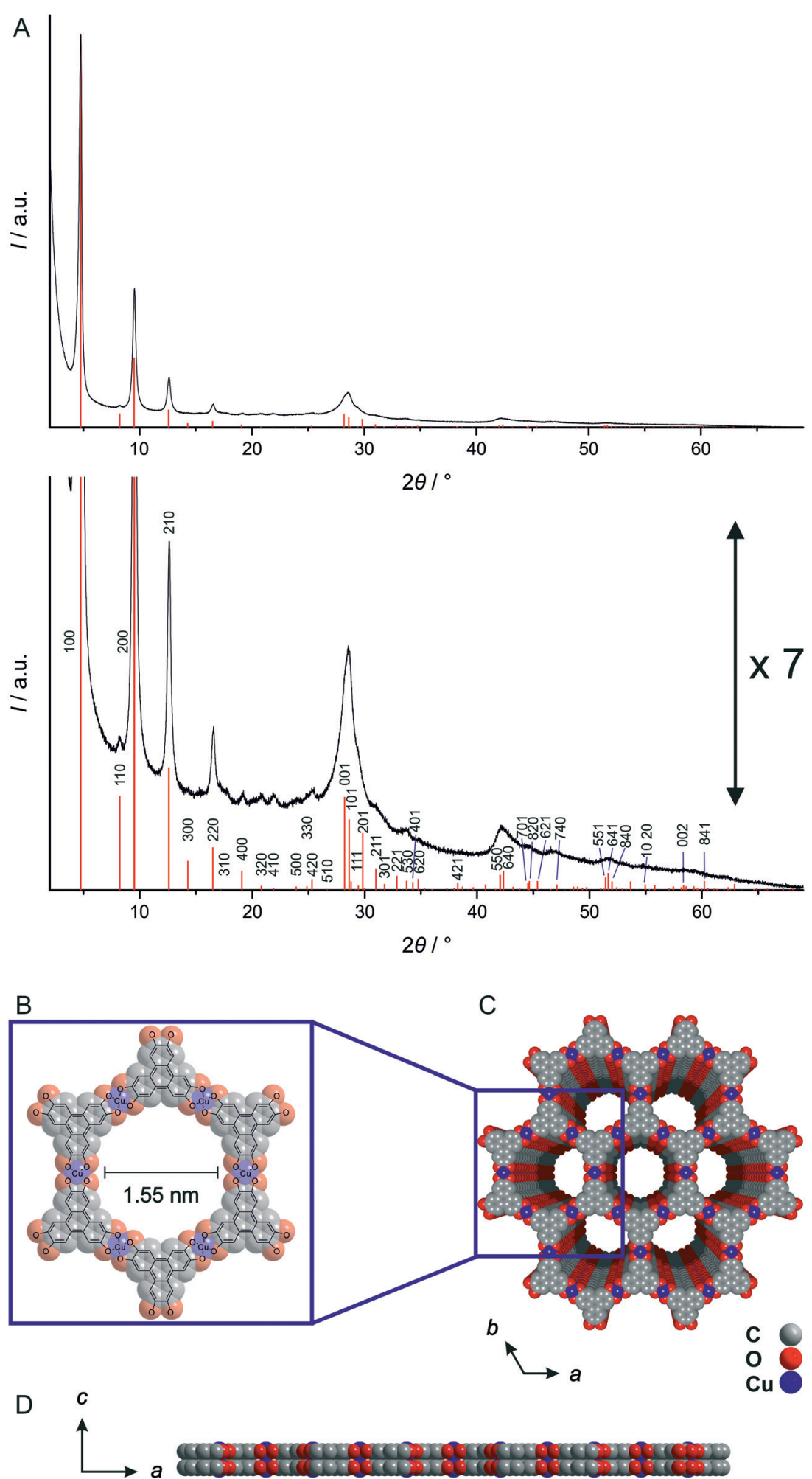

Fig. 1 A) Powder X-ray diffraction pattern of $\mathrm{Cu}_{3} h h t p_{2}$-MOF platelet crystals synthesized using 50 eq. ammonia modulation; positions and intensities of a simulated pattern are given as red lines. For better visibility of all peaks two versions of the pattern with different intensity scales are shown. The simulated XRD pattern is based on the structure shown in B, C and D; B) $a-b$ in-plane layer structure of $\mathrm{Cu}_{3} h h_{t} p_{2}-\mathrm{MOF} ; \mathrm{C}$ and $\mathrm{D}$ ) stacking of the layers along the $c$ direction in an AA fashion. 
the measurement, a wet argon stream which had passed through water was used to achieve a water-saturated gas stream.

Sensing experiments with coatings were performed with coated glass slides $\left(2 \times 2 \mathrm{~cm}^{2}\right)$ in a laboratory quartz glass tube. Prior to the measurement the glass slides were mounted in a holder, bonded to copper electrodes with conductive silver ink and placed in the gas stream. A constant potential of $500 \mathrm{mV}$ was applied to the electrodes using an Ametek Princeton Applied Research Versastat 4 potentiostat. To remove adsorbed guests, especially adsorbed water, the setup was flushed with argon for at least 1 hour before the experiment. For the measurement, $20 \mu \mathrm{L}$ of $\mathrm{MeOH}$ was injected into the argon stream, using a syringe and employing a glass flask with a septum; the glass flask is placed in front of the quartz glass tube setup.

\section{Results and discussion}

\subsection{Structure simulation}

Single crystals and correspondingly single crystal structures of 2D layered materials are often difficult to obtain. ${ }^{36-38}$ Also for the graphene-like MOFs, true single crystals suitable for single crystal X-ray diffraction studies have not been obtained so far. Nevertheless, some valuable statements about the structure of the $\mathrm{Cu}_{3} h h t p_{2}$-MOF can be made by close inspection of the X-ray diffractogram, using a combination of simulations and arguments from the theory of diffraction of twodimensional materials. ${ }^{36,38,39}$ These statements are based on the X-ray diffraction pattern displayed in Fig. 1A which stems from a material of comparatively high crystallinity, consisting of platelet-like crystals and obtained within this work by modulation of the synthesis with $0.3 \mathrm{mmol}_{6}$ hhtph and 50 eq. ammonia (see below).

Fig. 1A shows the indexed experimental X-ray diffraction pattern together with diffraction lines calculated from a simulated model. The experimental $h k 0$ reflections, especially those in the $2 \theta$ region up to $c a .26^{\circ} 2 \theta$, are relatively sharp. Their positions and intensities show good agreement with the simulated diffraction data depicted as line diagram. This certifies the basic correctness of the structure within the $a, b$ plane (depicted in Fig. 1B) and indicates that within this plane, the degree of disorder is low. Also, there is no significant asymmetric broadening of the diffraction peaks and thus no indication for excessive disorder in the stacking of the basic layers. Reflections of the $h k l$ family which relate to this disorder are weak, but some can be discerned as distinct peaks in the shoulders of other reflections, e.g. (211) at $31.0^{\circ}$ $2 \theta$. The simulated line pattern was calculated on the basis of an AA stacking of the layers as depicted in Fig. 1C and D.

The first non- $h k 0$ reflection appears at $28.2^{\circ} 2 \theta$. It is the (001) reflection related to the order along the $c$ axis, i.e. perpendicular to the layers. Although it overlaps with the (101) reflection, it is obvious that this reflection is considerably broadened. There are several possible reasons for such a broadening. First of all, it can be associated with Scherrer broadening caused by the small thickness of the particles produced by the ammonia-modulated synthesis used by us (see below), i.e. their short coherence length along the $c$ axis. Secondly, structural reasons are possible. Disorder of the stacking of the layers (translational or rotational) would lead to slightly varying interlayer distances with correspondingly varying $d_{001}$ distances; however, significant stacking disorder can be ruled out on the basis of the symmetric appearance of $h k 0$ reflections. It cannot be ruled out, however, that the layers are (irregularly) corrugated or curved which would also result in varying $d_{100}$ distances. The open and soft metal-organic framework is probably much more susceptible to such corrugation than more stiff layers, like, e.g., graphene layers.

From the position of the broadened 001 reflection, a mean interlayer distance of $3.16 \AA$ can be calculated. This value is considerably lower than in graphite and the values given for other related graphene-analogue $\mathrm{MOFs}^{7,8,11}$ and COFs. ${ }^{40}$ Probably, most of the structures described in the literature were not refined for this parameter. For example, the $\mathrm{Cu}_{3}$ hitp 2 -MOF described in ref. 8 seems to have a shorter interlayer distance than given by the presented structure simulation. In fact, the broad 001 reflection appears at slightly higher $2 \theta$ values in comparison to the calculated value of $27.8^{\circ} 2 \theta$ (representing an interlayer distance of $3.3 \AA$ ). ${ }^{7}$ The short interlayer distance observed on the $\mathrm{Cu}_{3} h h t p_{2}-\mathrm{MOF}$ might be indicative of attractive metal-metal interactions between the copper cations in adjacent layers which can occur in the proposed AA stacking.

Summarizing, the $\mathrm{Cu}_{3} h h t p_{2}$-MOF platelet crystals synthesized and inspected in this work appear to possess a high crystallinity, with no significant stacking or turbostratic disorder. The diffraction data are in agreement with an AA stacking which might be stabilized by attractive metal-metal interactions. The stacking is important as it defines the pore shape; for an AA stacking, unobtruded one-dimensional channels with a diameter of $1.55 \mathrm{~nm}$ result.

\subsection{Influence of additives on morphology}

In order to study the influence of additives on the crystal morphology of the $\mathrm{Cu}_{3} h h t p_{2}-\mathrm{MOF}$, we modified the synthesis described by Hmadeh et al. ${ }^{11}$ Reactions were carried out at $80^{\circ} \mathrm{C}$ in water. Instead of using copper(II) trifluoroacetylacetonate, with a rather strongly coordinating anion, as precursor, and NMP as additive, the synthesis was carried out with copper(II) nitrate trihydrate. We employed different additives in different amounts, among them alcohols, amines and azines as well as DMF and THF. The additives with a nitrogen-based ligand function were used because it was expected that they could coordinate to $\mathrm{Cu}^{2+}$ cations in a square-planar environment as present in the $\mathrm{Cu}_{3} h h t p_{2}$ layer structure and thus might effect a strong modulating action. Using coordination modulation, we hoped to reduce the amount of stacking of the layers and thereby suppress the crystal growth along the $c$-axis.

In general, the use of alcohols, DMF and THF leads to only very low yields of the MOF. The products consist mainly 
of unreacted $\mathrm{H}_{6} h h t p h$ linker. Using amines or pyridine, it is possible to receive the desired phase of the $\mathrm{Cu}_{3} h h t p_{2}$-MOF as the main product (Fig. 2). Probably, this is mainly caused by the improved solubility of the linker at higher $\mathrm{pH}$. The formation of elemental copper was observed when amines with longer alkyl chains were used. The best products with regard to crystallinity were received with pyridine and ammonia.

To further investigate the influence of the additives pyridine and ammonia, we used different concentrations of these modulators. All products showed a dark blue to black colour when dried. Except for the sample prepared with 10 equivalents of pyridine, which shows additional reflections assigned to unreacted linker molecules, the XRD patterns agree with phase-pure $\mathrm{Cu}_{3} h h t p_{2}$-MOF (Fig. $3 \mathrm{~A}$ and B). SEM images (Fig. 3C) show that the products from syntheses with pyridine do not show the desired crystal morphology. Especially with low amounts of pyridine, small agglomerated particles with uncharacteristic morphologies can be observed. This product bears some similarities to the product from the nonmodulated original synthesis, which consists of small rodlike, strongly aggregated particles. ${ }^{11}$ Only when 50 equivalents of pyridine were used during the synthesis, the product appears to have a more flake-like shape. In contrast, all samples synthesized in the presence of ammonia show a welldefined flake-like morphology. Individual platelets have a diameter of several micrometers and are randomly stacked on each other. Because of the very broad size distribution, it is hard to discern a trend in the particle diameter as a function of the amounts of ammonia employed during the synthesis. The thickness of individual flakes can be estimated from those particles in the SEM pictures which stand on an edge to be less than $20 \mathrm{~nm}$. This is in good agreement with the very broad 001 reflection at $28.2^{\circ} 2 \theta$. Especially the sample synthesized in the presence of 50 equivalents of ammonia

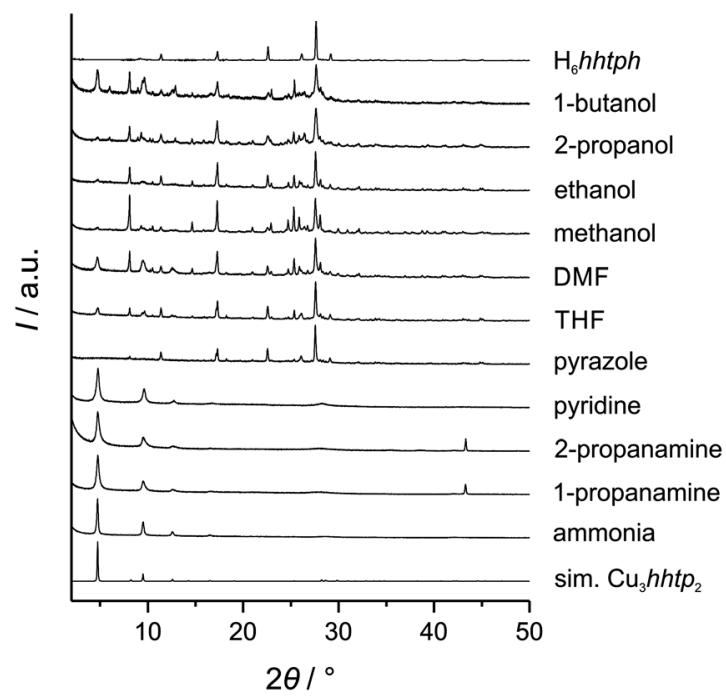

Fig. 2 Experimental XRD patterns of products prepared in the presence of different additives during the synthesis. 50 equivalents of the additive with respect to the $\mathrm{H}_{6} h h t p h$ linker amount were used. The reflection at $43.3^{\circ} 2 \theta$ stems from elemental copper. shows platelets with a regular morphology and clean surfaces. This result can be explained by coordination modulation, assuming that ammonia molecules bind at free coordination sites of $\mathrm{Cu}^{2+}$ ions which are in a square-planar environment of the hhtp ligands. For the growth of crystals along the $c$ direction, these ammonia ligands have to be removed first, explaining the small elongation of the crystals along this direction.

Apart from a coordination-modulation effect, the aminecontaining additives also increase the $\mathrm{pH}$ of the synthesis solution. To exclude this $\mathrm{pH}$ increase as a possible reason for the change in morphology we conducted further experiments where no amine-containing additives were added, but where the $\mathrm{pH}$ of the synthesis solution was adjusted to higher values by the addition of $\mathrm{NaOH}$. Investigation of the products by PXRD (see ESI $\dagger$ Fig. S6) and SEM (see ESI $\dagger$ Fig. S7) showed that no flake-like morphologies of the $\mathrm{Cu}_{3} h h t p_{2}$-MOF could be obtained in this way. At higher $\mathrm{pH}$ values some $\mathrm{Cu}_{2} \mathrm{O}$ is formed; at pH 14 the oxide is the major product according to PXRD. This is line with the observation that only ammonia and (to a lesser extent) pyridine exert a modulating effect, whereas pyrazole and 1- and 2-propanamine, which also increase the $\mathrm{pH}$, do not.

\subsection{Characterisation of platelet-like nanoparticles}

The thin platelets obtained from the ammonia-augmented synthesis are very interesting for several reasons. Firstly, within these platelets the pores run perpendicular to the basal plane, resulting in many short pores and many pore entrances; thus, the pore system can be easily accessed, diffusion paths are short, and sorption as well as desorption kinetics should be fast. Secondly, nanoparticles of defined morphology are well-suited precursors for various fabrication methods used to generate specific shapes and devices. Thirdly, the yield of this synthesis is easily scalable to at least $120 \mathrm{mg}$ per batch. Therefore, we set out to characterize these particles in more detail.

The nanoplatelets of the $\mathrm{Cu}_{3} h h t p_{2}$-MOF are easily dispersed in water; pH-dependent zeta potential measurements (Fig. 4A) reveal that the particles are negatively charged above the isoelectric point at $\mathrm{pH} 2$ and have a zeta potential below $-30 \mathrm{mV}$ above $\mathrm{pH}$ values of $c a$. 4.5. This explains the very high stability of dispersions at neutral or basic conditions.

SEM and AFM images (Fig. 4B and D) show thin particles with a non-uniform geometry and diameters extending up to a few micrometers. The thickness of these very thin particles, as determined by AFM height measurements, is about 7.5$10.4 \mathrm{~nm}$. Considering an interlayer distance of $0.316 \mathrm{~nm}$, this height corresponds to 24-33 stacked layers of $\mathrm{Cu}_{3} h h t p_{2}$. The hexagonal honeycomb pattern observed in TEM images (Fig. 4C) also confirms the orientation of the 1D pore system perpendicular to the surface of the particles. The determination of the pore size from the TEM picture is somewhat ambiguous because of the damage caused by the electron beam. Nevertheless, a pore size between 1.4 and $1.6 \mathrm{~nm}$ can be 

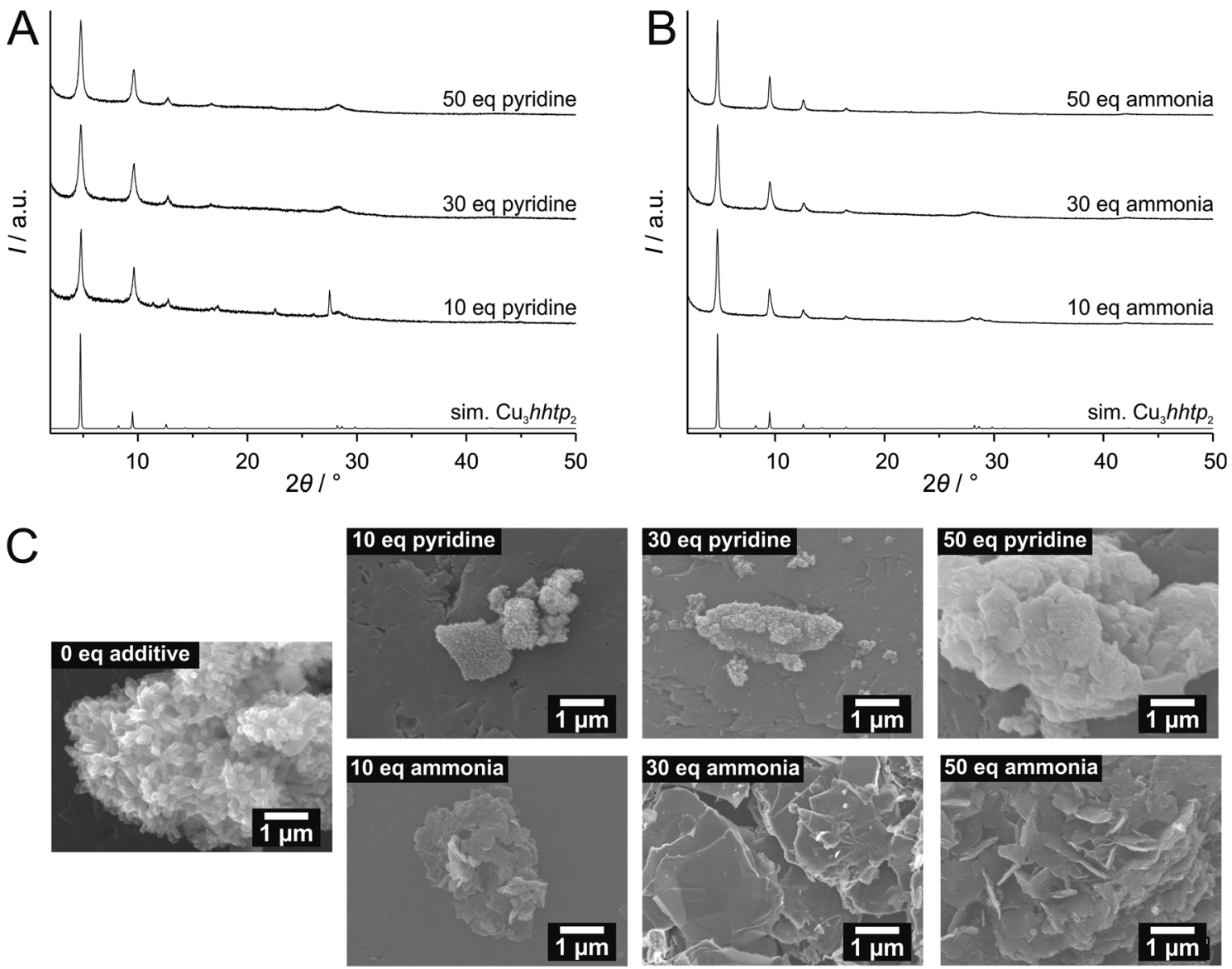

Fig. 3 (A) XRD patterns of the products from syntheses to which pyridine was added. (B) XRD patterns of the products from syntheses to which ammonia was added. In both B and C, the simulated XRD pattern of a layer of the $\mathrm{Cu}_{3} h \mathrm{htp}_{2}-\mathrm{MOF}$ is shown for comparison. (C) SEM images of the products received by syntheses to which pyridine or ammonia was added, respectively. For comparison, the product from a synthesis where no additive was used is also shown.

observed, which is in very good agreement with the crystallographic pore size of $1.55 \mathrm{~nm}$.

The diffractograms shown in Fig. 3B show no significant changes in the crystallinity with the amount of ammonia added during the synthesis. Therefore, we set out to also compare the sorption properties of the products of these syntheses and performed argon and water physisorption measurements to investigate the influence of added ammonia. The argon physisorption isotherms (Fig. 5A) show clearly that higher BET surface areas are obtained with higher amounts of added ammonia. The sample prepared with 50 equivalents ammonia exhibits a BET surface area of $512 \mathrm{~m}^{2} \mathrm{~g}^{-1}$. The pore size of ca. $1.6 \mathrm{~nm}$ (Fig. 5B), determined by NLDFT fitting of the sorption data, agrees well with the crystallographically expected value of $1.55 \mathrm{~nm}$. The small hysteresis in the mesopore regime $\left(p / p_{0}=0.1-0.9\right)$ as well as the strong increase of adsorbed volume at $p / p_{0}>0.9$ are probably caused by disordered interparticular pore volume.

In contrast to this, the sorption properties of water are less strongly affected by the amount of ammonia added during the synthesis, although (and as in the case of argon sorption), the sample prepared with 10 equivalents of ammonia shows the lowest uptake. On the other hand, the samples prepared with 30 and 50 equivalents exhibit very similar sorption properties with a maximum water uptake of $c a .40 \mathrm{wt} \%$. Water uptake starts at very small pressures. The material can thus be considered to be strongly hydrophilic.

To check the thermal stability of the $\mathrm{Cu}_{3} h h t p_{2}-\mathrm{MOF}$ coupled thermogravimetric, differential thermal analysis and mass spectroscopy measurements were performed on a Soxhlet-extracted sample which was prepared in the presence of 50 equivalents ammonia (Fig. 6). The experiment was carried out in an oxygen/argon (20/80) atmosphere. The first step is correlated to the evaporation of guest molecules from the framework. These guests are adsorbed water (or remaining ammonia), as confirmed by the signal for $m / z 17$ in mass spectroscopy. Above $80^{\circ} \mathrm{C}$, organic compounds start to slowly combust in an exothermal reaction. This value is lower than the reported thermal stabilities of the related MOFs $\mathrm{Ni}_{3} h i t p_{2}$ $\left(300{ }^{\circ} \mathrm{C}\right),{ }^{8} \mathrm{Co}_{3} h h t p_{2}\left(250{ }^{\circ} \mathrm{C}\right)^{11}$ and $\mathrm{Ni}_{3} h h t p_{2}\left(200{ }^{\circ} \mathrm{C}\right) \cdot{ }^{11}$ The mass signal of 28 can be related to $\mathrm{CO}_{2}{ }^{2+}$ or $\mathrm{C}_{2} \mathrm{H}_{4}{ }^{+\cdot}$ species and derives most probably from organic parts of the framework. Additionally, $\mathrm{m} / \mathrm{z} 12$ is related to $\mathrm{C}^{+}$species which can also be associated with the combustion of organic 

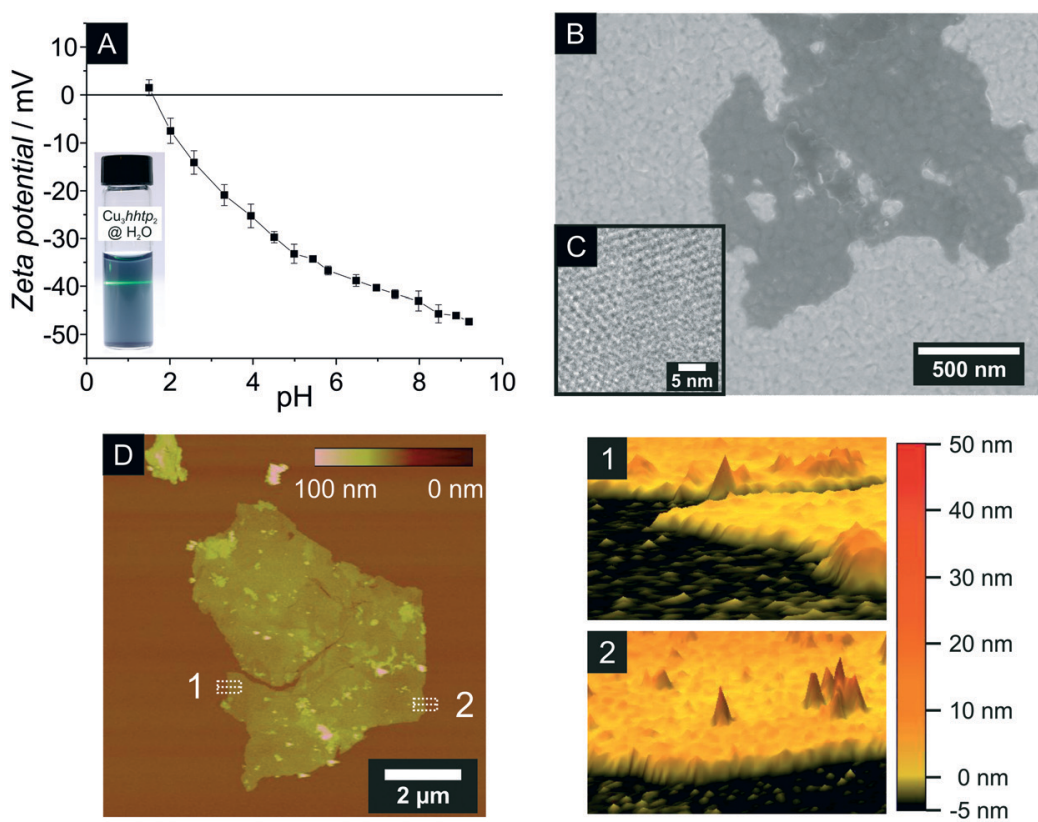

Position 1

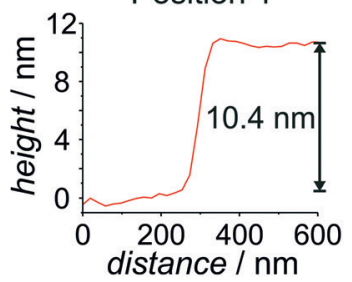

Position 2

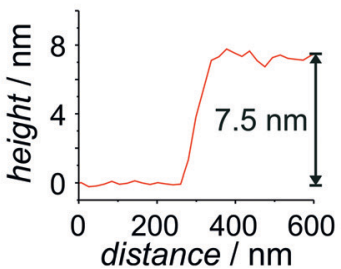

Fig. 4 Characterisation of nanoplatelets of the $\mathrm{Cu}_{3} h \mathrm{htp}_{2}$-MOF. (A) Zeta potential measurement and photography of a stable colloidal dispersion of $\mathrm{Cu}_{3} h h t p_{2}$ in water $\left(2.4 \mathrm{mg} \mathrm{mL}^{-1}\right)$ with a crossing laser beam. (B) HR-SEM image of $\mathrm{Cu}_{3} h h \mathrm{Hp}_{2}$ nanoplatelets on a gold-coated Si wafer; note that the substrate surface can be seen through the flaky material. (C) TEM image confirming the perpendicular orientation of the hexagonal pore system to the particle surface; pore sizes can be estimated to ca. 1.4-1.6 nm. (D) Left: AFM image of the topography of thin flakes of Cuzhtp ${ }_{2}$ prepared from the dispersion shown in $\mathrm{A}$ by drop-casting on an $\mathrm{SiO}_{2} / \mathrm{Si}$ wafer; right: three-dimensional views of the areas marked as 1 and 2 in the topographical image; bottom: averaged height profiles of these areas.

compounds. Combustion intensifies above $200{ }^{\circ} \mathrm{C}$ and is completed at $350{ }^{\circ} \mathrm{C}$. The residue was proven to be $\mathrm{CuO}$ by XRD. Subtracting the 3 mass-\% of guest species liberated in the first step, the amount of organic components corresponds to 73.1 mass- $\%$ and the $\mathrm{CuO}$ residue to 26.9 mass- $\%$; this fits very well to the theoretical values of 72.7 mass-\% organic and 27.3 mass-\% CuO. Further investigations on the initial reaction of the framework to heating, carried out by XRD (Fig. 6C), prove the thermal stability of $\mathrm{Cu}_{3} h h t p_{2}$ up to $80{ }^{\circ} \mathrm{C}$. Upon further heating to $200{ }^{\circ} \mathrm{C}$, only amorphous material can be observed.

\subsection{Spray-coating of thin films}

For many envisaged applications of MOFs, processing and shaping of the material is a prerequisite. Nanoparticles with uniform morphology lend themselves for many controlled shaping processes. To demonstrate the advantages of the $\mathrm{Cu}_{3} h h t p_{2}$-MOF nanoplatelets described in this work, we chose to describe the generation of thin films by simple spraycoating procedures from water-based dispersions. We used an automated setup and added the surfactant $\operatorname{Span}{ }^{\circledR} 80$ in order to obtain crack-free and homogeneous coatings. As substrates we used glass slides and polymer foils.

Fig. 7 depicts the results for the spray-coating experiments on glass slides. Fig. 7A shows a homogeneously covered glass substrate with the typical dark blue colour. SEM pictures show a complete and crack-free covering of the substrate (Fig. 7B). From SEM cross-sections as the one depicted in Fig. 7C, layer thicknesses can be determined. These range from $1 \mu \mathrm{m}$ to $3 \mu \mathrm{m}$, depending on the number of coating cycles (50-100). In both SEM images, a strong texture of the nanoplatelets can be observed. With a strong preference, the flakes are oriented parallel to the substrate surface. Also, the nanoparticles are obviously in close contact. The strong preferred orientation of the $\mathrm{Cu}_{3} h h t p_{2}$ particles was confirmed by XRD measurements (Fig. 7D). In comparison to the bulk material, the relative intensity of the (001) reflection is strongly increased and the other reflections are virtually absent. This is not due to a degradation of the material: powder samples received from these coatings still show the characteristic XRD pattern of the $\mathrm{Cu}_{3} h h t p_{2}-\mathrm{MOF}$ and are again comparable to the 

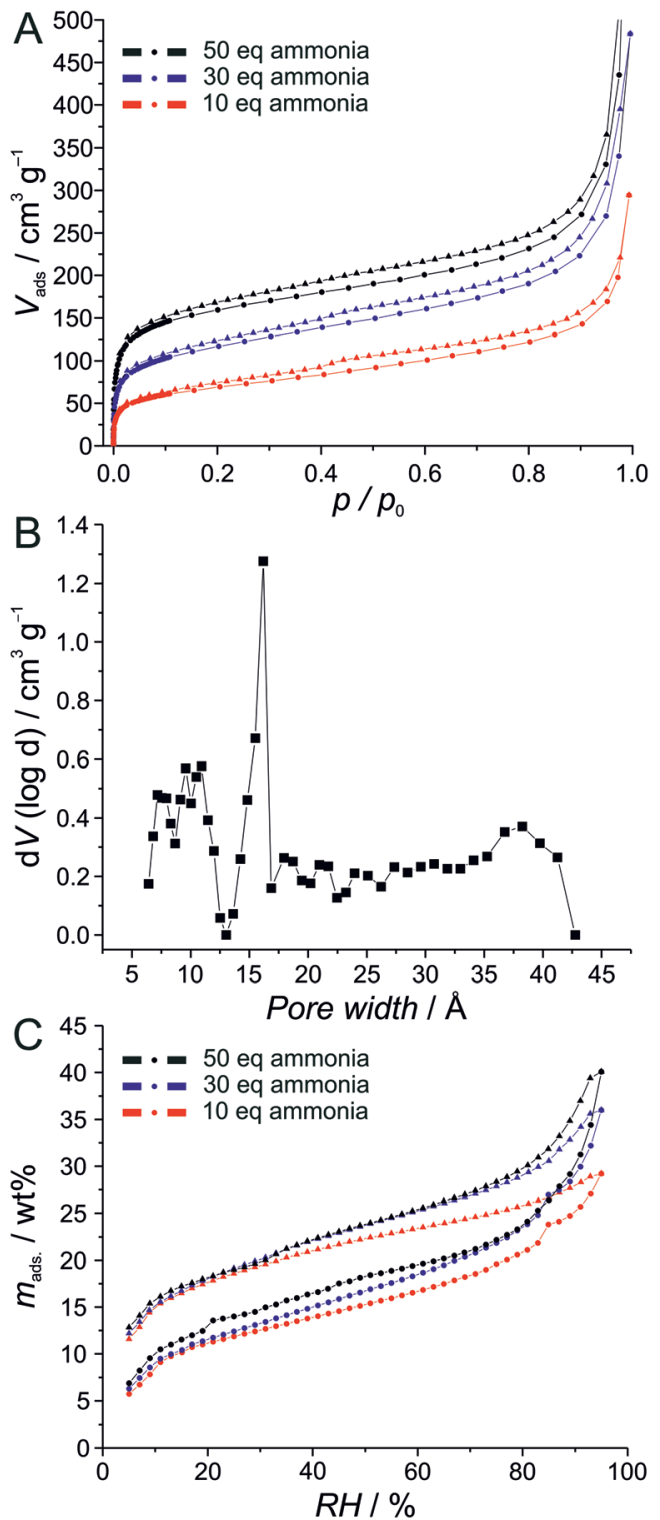

Fig. 5 Investigation of the sorption properties of nanoplatelets of the $\mathrm{Cu}_{3} h h t p_{2}$-MOF prepared in the presence of different amounts of ammonia; circles represent adsorption, triangles represent desorption. (A) Argon physisorption isotherms. (B) Pore size distribution derived from the argon adsorption data of the sample prepared with 50 equivalent of ammonia. (C) Water physisorption isotherms.

bulk sample. The preferred orientation, with the conducting planes all lying parallel, and the good contacts between the particles, both promise favourable pathways for charge carriers and thus should support high electrical conductivity. Additionally, with the preferred orientation of the primary particles, the pore channels are arranged perpendicular to the coating surface. This in turn leads to an easily accessible pore system as it is preferred in applications like sensing, leading to strong and fast responses.

To extend the range of possible applications of our $\mathrm{Cu}_{3} h h t p_{2}$-MOF nanoplatelets to flexible electronics and also to show the scalability of our approach to larger samples, we
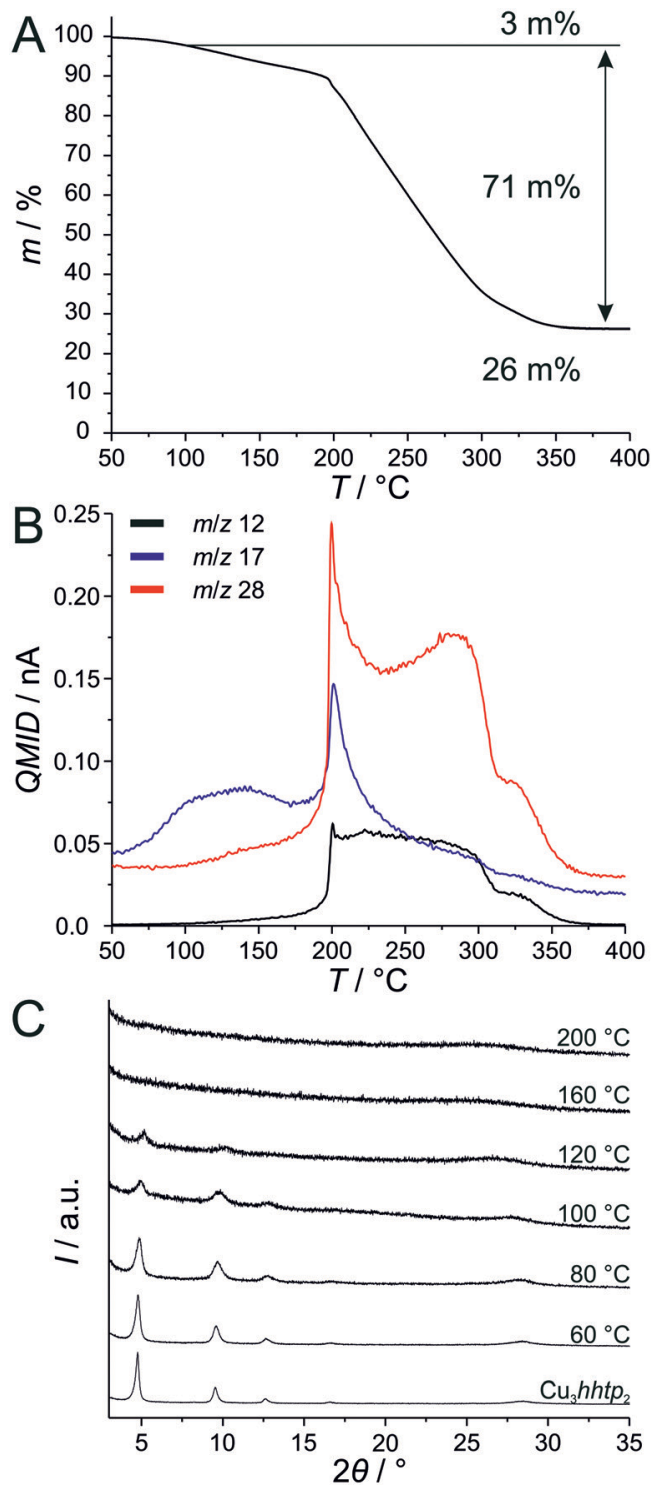

Fig. 6 Investigation of the thermal properties of nanoplatelets of the $\mathrm{Cu}_{3} h h$ tp $_{2}$-MOF; the sample was prepared in the presence of 50 equivalents of ammonia and Soxhlet-extracted. A) Thermogravimetric measurement with (B) simultaneously recorded mass spectroscopic data. C) X-ray powder diffraction patterns obtained after heating samples to the indicated temperatures for 24 hours in air.

used thin polycarbonate foils as substrates. By the use of different stencils to generate patterned conducting pathways, we are also able to further demonstrate the flexibility of this method. Using the automated spray-coating setup and large polycarbonate foils in conjunction with these stencils, we fabricated the specimen shown in Fig. 8. The maximum coating area is only limited by the dimension of the automated spray-coating device. Additionally, very small and fine structures or even switch board circuits paths can be generated without adapting the coating parameters. The same coating quality is obtained in all cases (see ESI $\uparrow$ Fig. S1D-G). It should be noted that coatings on polycarbonate without using a surfactant are not nearly as homogeneous. 

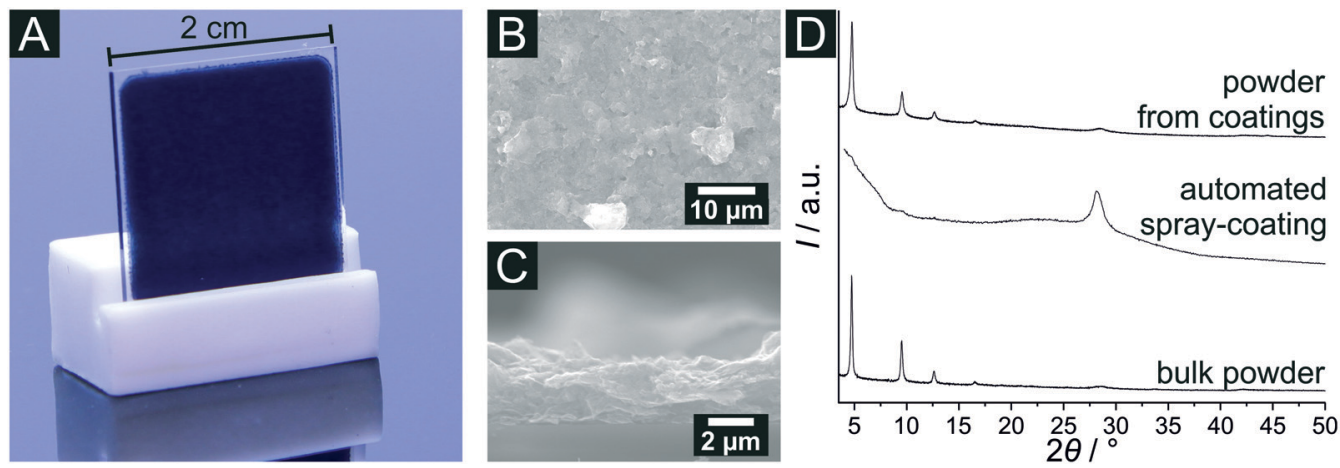

Fig. 7 Characterisation of spray-coated thin films of the $\mathrm{Cu}_{3} h h t p_{2}-\mathrm{MOF}$ on glass slides, obtained by automated spray coating with Span ${ }^{8} 80$ as surfactant. (A) Photography of a spray-coated layer. (B) SEM image of the surface of the coating. (C) Cross-section of the coating, showing a rough surface and a thickness of about $2.7 \mu \mathrm{m}$ for this sample. (D) XRD patterns comparing a bulk powder sample of the $\mathrm{Cu}_{3} h \mathrm{Ctp}_{2}-\mathrm{MOF}$ after Soxhlet extraction, of a spray-coated film, and of a powder re-isolated from the coating.
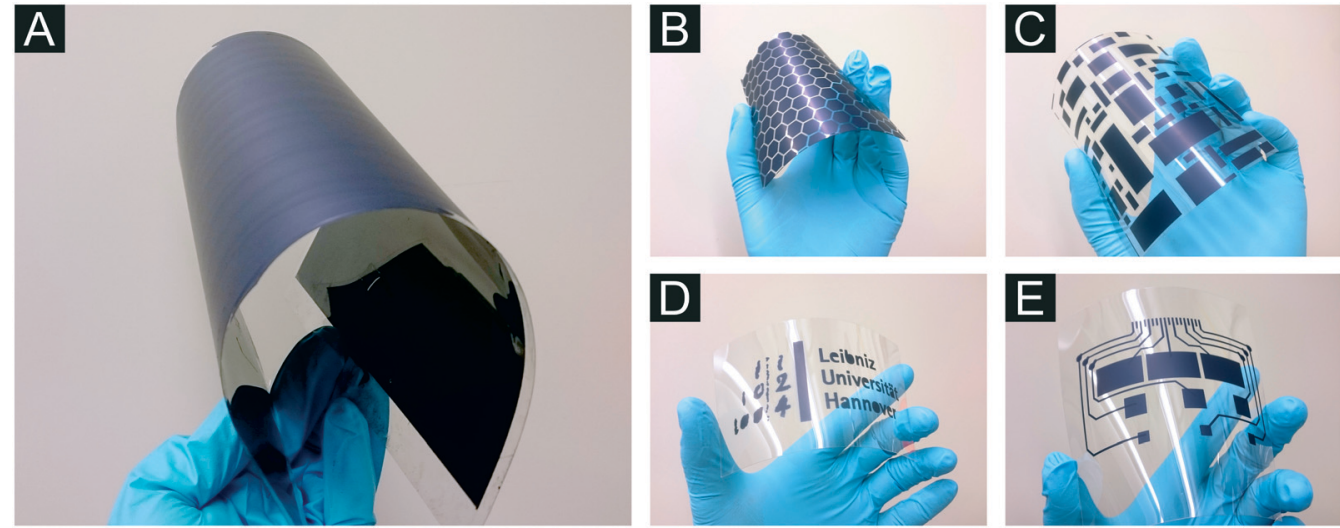

Fig. 8 Photographies of spray-coated thin films of the $\mathrm{Cu}_{3} h h t p_{2}-\mathrm{MOF}$ on polycarbonate foils. Fifty spraying cycles were used in each experiment. A: Large scale homogenous coatings (on DIN A4 sheet) adhering very well to the polycarbonate substrate even under strong bending are obtained. B-E: Different patterns of the MOF can be generated using corresponding stencils; image E shows a typical circuit used for switch boards.

\subsection{Electrical and electronic properties}

The electrical properties of the $\mathrm{Cu}_{3} h h t p_{2}$-MOF nanoplatelet material were measured on bulk materials as pressed pellets of Soxhlet-extracted samples and on thin film coatings described in the preceding section. The pellets were measured using the van der Pauw setup (Fig. 9A) and give a value for the electrical conductivity of $0.045 \mathrm{~S} \mathrm{~cm}^{-1}$. This value surpasses all reported measurements of thin films ${ }^{19,20}$ or pressed pellets ${ }^{6}$ of the $\mathrm{Cu}_{3} h h t p_{2}$-MOF thus far; for a specimen denoted as single crystal, ${ }^{11}$ a value of $0.2 \mathrm{~S} \mathrm{~cm}^{-1}$ was reported.

The electrical conductivity of coatings produced from our nanoplatelets on glass and polycarbonate show enhanced electric conductivities of about $0.023 \mathrm{~S} \mathrm{~cm}^{-1}$ at room temperature. This is in a good agreement with recently reported results of coatings produced with a layer-by-layer method ${ }^{19}$ and surpasses the values of coatings produced at the liquidliquid interface. ${ }^{20}$ In contrast to these approaches, with automated spray-coating we can easily increase the coating area
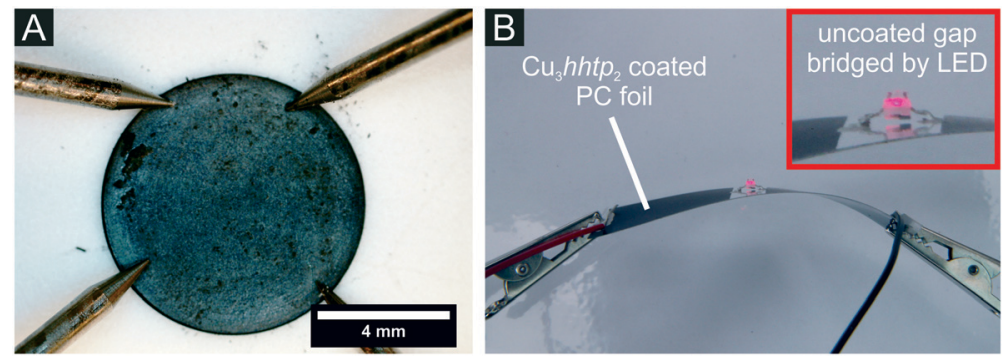

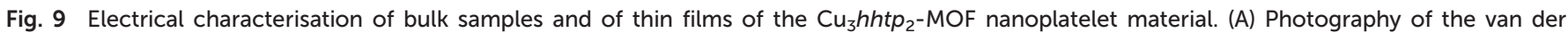
Pauw setup for pressed pellet measurements. (B) Coated polycarbonate foil, which is twisted and bent, bridged by a commercial LED emitting light after applying current to the system; inset showing higher magnification of the uncoated area with the LED. 


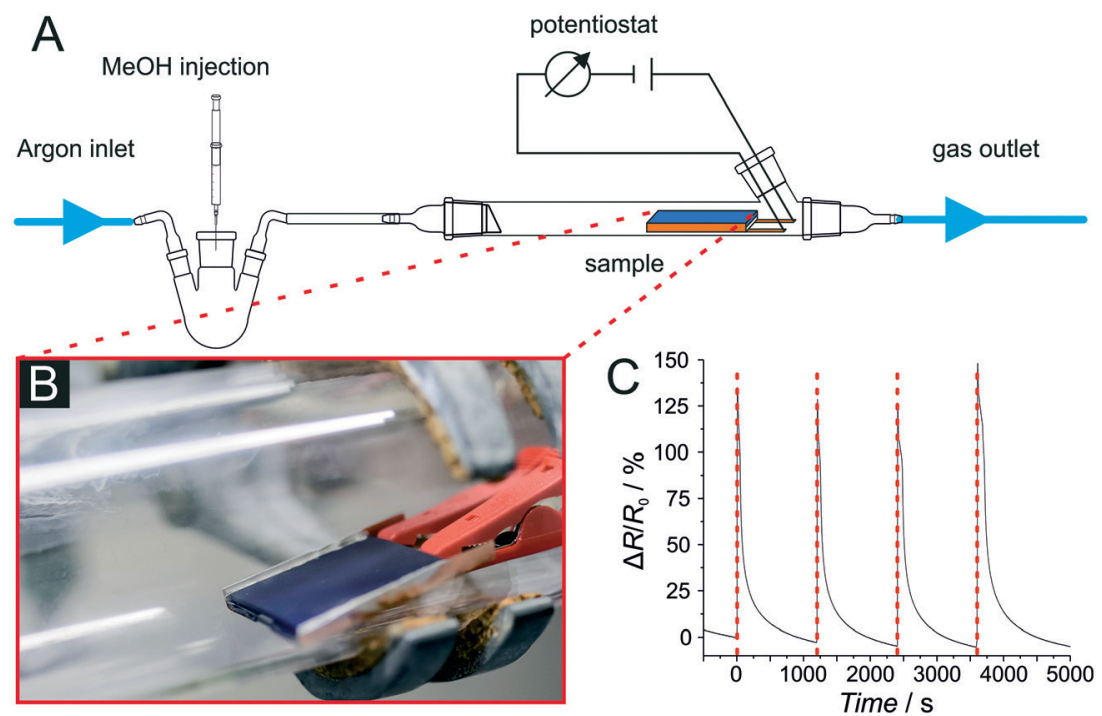

Fig. 10 Sensing experiments with $\mathrm{Cu}_{3} h \mathrm{htp}_{2}$-MOF-based coatings prepared from the nanoplatelet dispersion described in this work using airbrush spraying. (A) Setup of the experiment with a photography of the coated glass slide. (B) Sensing experiment with methanol. (C) Measured changes of resistance of the system coincide with the injection time points (red lines), showing very fast and reversible response to methanol in a passing gas stream.

and retain the good quality of the products. To proof the usability of our coatings in flexible electronics, we show in Fig. 9B a shining LED which is contacted by $\mathrm{Cu}_{3} h h t p_{2}-\mathrm{MOF}$ coatings on polycarbonate; this setup even works when the foil is twisted and bent.

By carrying out temperature-dependent measurements of the electrical conductivity, further information like the activation energy and the fundamental band gap can be gathered (see ESI $\dagger$ Fig. S2). The low thermal activation energy of $E_{\mathrm{A}}=$ $0.15 \mathrm{eV}$ and the band gap of $E_{\mathrm{g}}=0.301 \mathrm{eV}$ reveal good prerequisites for the electrical conductivity of the material. In addition, the activation energy is lower here in comparison to $E_{\mathrm{A}}=0.24 \mathrm{eV}$ measured on samples prepared by liquid-liquid interface synthesis. $^{20}$

XPS measurements (see ESI† Fig. S3) carried out on Soxhlet-extracted coatings on gold substrates show no hints of remaining ammonia or other nitrogen species in the sample. In agreement with recently reported measurements ${ }^{20}$ we can confirm, that only $\mathrm{Cu}^{2+}$ species can be detected. This is in line with the assumption that for neutral $\mathrm{Cu}_{3} h h t p_{2}$ the triphenylene linker molecule is in a semichinon state ${ }^{41}$ with a loosely bound radical.

\subsection{Sensing applications}

The novel materials described in this paper, namely the nanoplatelets of $\mathrm{Cu}_{3} h h t p_{2}$-MOF and the coatings derived therefrom, have several properties which favour their application in electric devices, as, e.g., sensors or supercapacitors. These assets are high electrical conductivity, an easily accessible pore system and facile processing for the fabrication of device-suited shapes. Here, we tested the usefulness of this material in sensing applications.
Water sorption measurement had shown that the $\mathrm{Cu}_{3} h h t p_{2}$-MOF is rather hydrophilic. As many sensing experiments are influenced by cross-sensitivity to water, we first of all tested the effect of water on the conductivity of this material. For this purpose, a water-saturated argon stream was passed through a measurement cell where a pellet of the MOF is placed between two brass electrodes; a constant potential of $500 \mathrm{mV}$ was applied to the system. Under dry conditions, an Ohmic behaviour of the system can be observed. When a water-saturated stream of argon is passed through the cell, the uptake of water during this process leads to a very strong increase in resistance up to $370 \%$ (see ESI $\dagger$ Fig. S4). A strong influence of water on the output signal of a sensing device relying on $\mathrm{Cu}_{3} h h t p_{2}$-MOF has therefore to be taken into account.

For preliminary investigations on the sensing properties of $\mathrm{Cu}_{3} h h t p_{2}$-MOF-based coatings, we placed coated glass slides in a simple sensing setup (Fig. 10). Using this setup, we observed very fast responses to methanol in the gas phase. The process is also reversible. The observed increase in resistance can be cycled reproducibly many times. Similar results were obtained with coated polycarbonate foils (see ESI $\uparrow$ Fig. S5), showing the suitability also for flexible sensing devices. Note also that the signal intensities remain constant (which is also true for further cycles) so that we can conclude that the material is stable under the conditions of the sensing setup. Nevertheless, in addition we exposed the material also to liquid methanol for $24 \mathrm{~h}$. Under these much harsher conditions, some degradation can be observed (see ESI $\dagger$ Fig. S8). Nevertheless, we can verify that the coatings produced from the nanoplatelet dispersion are very attractive for sensing applications. 


\section{Conclusions}

In this work we investigated the influence of additives used within a water-based synthesis system for the preparation of $\mathrm{Cu}_{3} h h t p_{2}$-MOF on the crystallinity and morphology of the products. With ammonia acting as a coordination modulator, the $\mathrm{Cu}_{3} h h t p_{2}$-MOF can be synthesized phase-pure and with a flake-like morphology on a large scale. These nanoparticles were thoroughly characterized. Conductivity measurements on pressed pellets reveal a conductivity of $0.045 \mathrm{~S} \mathrm{~cm}^{-1}$, an enhanced value in comparison to recent reports. ${ }^{6,19,20}$ Noteworthy, we find that the thermal stability of the $\mathrm{Cu}_{3} h h t p_{2}$ MOF is limited to $c a .80{ }^{\circ} \mathrm{C}$ in synthetic air $\left(\mathrm{O}_{2} / \mathrm{Ar}\right.$ : $\left.20 / 80\right)$. The flakes or platelets have diameters in the micrometer range and very small thicknesses of about 7.5-10.4 nm, corresponding to $c a$. 24-33 stacked layers. These nanoplatelets form very stable dispersions in water, opening interesting pathways for fabrication and shaping.

Using these aqueous dispersions, coatings of the novel nanoplatelets were prepared on glass slides and on polycarbonate foils by spray-coating, allowing also patterned deposition of the MOF coatings. Several properties of these coatings make them promising for sensing applications: i. good interparticle contacts, leading to enhanced electrical conductivity; ii. high degree of texture within the coatings with the nanoplatelets lying parallel to the substrate surface, thus exposing many pore entrances offering short diffusion pathways. In sensors, these properties can lead to well-defined signals and fast response and regeneration times. The sensing behaviour of the coatings was tested in a preliminary set-up. Upon offering methanol in a gas stream, reproducible signals with fast response and recovery were observed.

In conclusion, this work shows that apart from the composition and the structure of a MOF, crystal morphology is an important parameter with regard to shaping a MOF for a certain application. We expect the nanoplatelets and the coatings described here to be also suitable for other applications in MOF-based flexible electronic devices.

\section{Conflicts of interest}

There are no conflicts to declare.

\section{Acknowledgements}

The TEM investigations were performed at the Laboratory of Nano and Quantum Engineering (LNQE). The XPS investigations were performed at the Institut für Festkörperphysik of the Leibniz University Hannover (Group Prof. H. Pfnür).

\section{Notes and references}

1 A. A. Talin, A. Centrone, A. C. Ford, M. E. Foster, V. Stavila, P. Haney, R. A. Kinney, V. Szalai, F. El Gabaly, H. P. Yoon, F. Léonard and M. D. Allendorf, Science, 2013, 343, 66.

2 T. C. Wang, I. Hod, C. O. Audu, N. A. Vermeulen and S. T. Nguyen, ACS Appl. Mater. Interfaces, 2017, 9, 12584.
3 R. Sakamoto, T. Kambe, S. Tsukada, K. Takada, K. Hoshiko, Y. Kitagawa, M. Okumura and H. Nishihara, Inorg. Chem., 2013, 52, 7411-7416.

4 X. Huang, P. Sheng, Z. Tu, F. Zhang, J. Wang, H. Geng, Y. Zou, C. Di, Y. Yi, Y. Sun, W. Xu and D. Zhu, Nat. Commun., 2015, 6, 7408.

5 A. J. Clough, J. W. Yoo, M. H. Mecklenburg and S. C. Marinescu, J. Am. Chem. Soc., 2015, 137, 118-121.

6 M. G. Campbell, S. F. Liu, T. M. Swager and M. Dincă, J. Am. Chem. Soc., 2015, 137, 13780-13783.

7 M. G. Campbell, D. Sheberla, S. F. Liu, T. M. Swager and M. Dincă, Angew. Chem., Int. Ed., 2015, 54, 4349-4352.

8 D. Sheberla, L. Sun, M. A. Blood-Forsythe, S. Er, C. R. Wade, C. K. Brozek, A. Aspuru-Guzik and M. Dincă, J. Am. Chem. Soc., 2014, 136, 8859-8862.

9 D. Sheberla, J. C. Bachman, J. S. Elias, C.-J. Sun, Y. ShaoHorn and M. Dincă, Nat. Mater., 2017, 16, 220-224.

10 J. Cui and Z. Xu, Chem. Commun., 2014, 50, 3986-3988.

11 M. Hmadeh, Z. Lu, Z. Liu, F. Gándara, H. Furukawa, S. Wan, V. Augustyn, R. Chang, L. Liao, F. Zhou, E. Perre, V. Ozolins, K. Suenaga, X. Duan, B. Dunn, Y. Yamamto, O. Terasaki and O. M. Yaghi, Chem. Mater., 2012, 24, 3511-3513.

12 L. Sun, B. Liao, D. Sheberla, D. Kraemer, J. Zhou, E. A. Stach, D. Zakharov, V. Stavila, A. A. Talin, Y. Ge, M. D. Allendorf, G. Chen, F. Léonard and M. Dincă, Joule, 2017, 1, 168-177.

13 R. Dong, M. Pfeffermann, H. Liang, Z. Zheng, X. Zhu, J. Zhang and X. Feng, Angew. Chem., Int. Ed., 2015, 54, 12058-12063.

14 L. Wang, D. C. Tranca, J. Zhang, Y. Qi, S. Sfaelou, T. Zhang, R. Dong, X. Zhuang, Z. Zheng and G. Seifert, Small, 2017, 13, $1-8$.

15 E. M. Miner, T. Fukushima, D. Sheberla, L. Sun, Y. Surendranath and M. Dincă, Nat. Commun., 2016, 7, 10942.

16 D. Y. Tie and Z. Chen, RSC Adv., 2015, 5, 55186-55190.

17 S. Chen, J. Dai and X. C. Zeng, Phys. Chem. Chem. Phys., 2015, 17, 5954-5958.

18 M. E. Foster, K. Sohlberg, C. D. Spataru and M. D. Allendorf, J. Phys. Chem. C, 2016, 120, 15001-15008.

19 M.-S. Yao, X.-J. Lv, Z.-H. Fu, W.-H. Li, W.-H. Deng, G.-D. $\mathrm{Wu}$ and $\mathrm{G} . \mathrm{Xu}$, Angew. Chem., Int. Ed., 2017, 3, 16510-16514.

20 V. Rubio-Giménez, M. Galbiati, J. Castells-Gil, N. AlmoraBarrios, J. Navarro-Sánchez, G. Escorcia-Ariza, M. Mattera, T. Arnold, J. Rawle, S. Tatay, E. Coronado and C. MartíGastaldo, Adv. Mater., 2018, 30, 1704291.

21 D. Feng, T. Lei, M. R. Lukatskaya, J. Park, Z. Huang, M. Lee, L. Shaw, S. Chen, A. A. Yakovenko, A. Kulkarni, J. Xiao, K. Fredrickson, J. B. Tok, X. Zou, Y. Cui and Z. Bao, Nat. Energy, 2018, 3, 30-36.

22 J. H. Dou, L. Sun, Y. Ge, W. Li, C. H. Hendon, J. Li, S. Gul, J. Yano, E. A. Stach and M. Dincă, J. Am. Chem. Soc., 2017, 139, 13608-13611.

23 T. Tsuruoka, S. Furukawa, Y. Takashima, K. Yoshida, S. Isoda and S. Kitagawa, Angew. Chem., Int. Ed., 2009, 48, 4739-4743. 
24 S. Diring, S. Furukawa, Y. Takashima, T. Tsuruoka and S. Kitagawa, Chem. Mater., 2010, 22, 4531-4538.

25 A. Umemura, S. Diring, S. Furukawa, H. Uehara, T. Tsuruoka and S. Kitagawa, J. Am. Chem. Soc., 2011, 133, 15506-15513.

26 G. Zahn, P. Zerner, J. Lippke, F. L. Kempf, S. Lilienthal, C. A. Schröder, A. M. Schneider and P. Behrens, CrystEngComm, 2014, 16, 9198-9207.

27 A. Schaate, P. Roy, A. Godt, J. Lippke, F. Waltz, M. Wiebcke and P. Behrens, Chem. - Eur. J., 2011, 17, 6643-6651.

28 O. V. Gutov, S. Molina, E. C. Escudero-Adán and A. Shafir, Chem. - Eur. J., 2016, 22, 13582-13587.

29 G. Zahn, H. A. Schulze, J. Lippke, S. König, U. Sazama, M. Fröba and P. Behrens, Microporous Mesoporous Mater., 2015, 203, 186-194.

30 A. Schaate, S. Dühnen, G. Platz, S. Lilienthal, A. M. Schneider and P. Behrens, Eur. J. Inorg. Chem., 2012, 790-796.

31 M. E. Schweinefuß, S. Springer, I. A. Baburin, T. Hikov, K. Huber, S. Leoni and M. Wiebcke, Dalton Trans., 2014, 43, 3528-3536.
32 S. Springer, I. A. Baburin, T. Heinemeyer, J. G. Schiffmann, L. van Wüllen, S. Leoni and M. Wiebcke, CrystEngComm, 2016, 18, 2477-2489.

33 S. Springer, A. Satalov, J. Lippke and M. Wiebcke, Microporous Mesoporous Mater., 2014, 216, 161-170.

34 L. J. van der Pauw, Philips Tech. Rev., 1958, 20, 220-224.

35 A. A. Ramadan, R. D. Gould and A. Ashour, Thin Solid Films, 1994, 239, 272-275.

36 V. A. Drits and C. Tchoubar, X-Ray Diffraction by Disordered Lamellar Structures, Springer, Berlin Heidelberg, 1990.

37 M. Fröba, P. Behrens, H. P. Eickhoff and W. Metz, Carbon, 1991, 29, 909-913.

38 Z. Q. Li, C. J. Lu, Z. P. Xia, Y. Zhou and Z. Luo, Carbon, 2007, 45, 1686-1695.

39 H. Jagodzinski, Acta Crystallogr., 1949, 2, 201-207.

40 A. P. Côté, A. I. Benin, N. W. Ockwig, M. O'Keeffe, A. J. Matzger and O. M. Yaghi, Science, 2005, 310, 1166-1170.

41 C. S. Grange, A. J. H. M. Meijer and M. D. Ward, Dalton Trans., 2010, 39, 200-211. 